\title{
Double-Bounded Homotopy for Analysing Nonlinear Resistive Circuits
}

\author{
Héctor Vázquez-Leal, Luis Hernández-Martínez and Arturo Sarmiento-Reyes \\ National Institute for Astrophysics, Optics and Electronics \\ Electronics Department, CAD Group, Puebla, Mexico \\ E-mail hleal@inaoep.mx
}

\begin{abstract}
A homotopy method for obtaining DC solutions of nonlinear circuits is proposed. The homotopy method is called double-bounded homotopy and it is used to find multiple DC solutions. This method presents a novel stop criterion which is based on the property of tracing a double bounded trajectory. The main properties of the homotopy are explained by using the Lambert- $\mathcal{W}$ function.
\end{abstract}

\section{INTRODUCTION}

The problem of finding the DC operating point is important because it constitutes the starting point for other kinds of analyses such as the ac small-signal and transient analyses [1]. It consists in finding the roots of the nonlinear algebraic equations (NAEs) emanating from nonlinear resistive networks, NAEs that have the general form given by:

$$
\vec{f}(\vec{x})=\overrightarrow{0}
$$

where $\vec{x}$ are the unknowns accordingly to the circuit analysis method used to set-up the equilibrium equation of the circuit.

As it is well-known, equation (1) may posses a single solution, no solution at all, or multiple solutions. In this paper, the attention is focused on the last case, where most of the Newton-Raphson (NR) methods fail to converge. Homotopy methods have been considered as a good choice of methods that overcome the shortcomings of the NR-like methods [2], [3], [4].

Homotopy methods are based on stablishing an auxiliary equation in order to convert the problem of finding the roots of a NAE (a static problem) into a problem of finding the solution of an associated ordinary differential equation (a dynamic problem) [1], [5]. This auxiliary equation, also called the homotopy equation, is formed by adding a parameter to the original equation:

$$
\vec{H}(\vec{f}(\vec{x}), \lambda)=\overrightarrow{0}
$$

where $\vec{H}(\cdot)$ is the homotopy relationship, $\vec{f}(\vec{x})$ is the original equilibrium equation of the circuit, and $\lambda$ is the homotopy parameter. The solution is found by following a numeric integration procedure, where $\lambda$ is used as the integration parameter that varies from an initial value $\lambda_{0}$ to a value $\lambda^{*}$ where a solution $\vec{x}^{*}$, to the original system (1) is found. From this point, the integration procedure continues in order to search for another solution. The whole procedure results in tracing a path where the solutions lie.
Although, homotopy methods are able to find more than one solution to (1), they still exhibit several lacks. Among them, it is worthy to mention:

- No global convergence. The capability of finding all solutions cannot be always guaranteed. In fact, this depends on a series of issues, such as the initial point where the homotopy starts to trace the solution, the trace method and the type of nonlinear equation.

- Stop criterion. There are two types of paths of solutions, open and closed paths, and spite the type of path the main problem is when to stop searching for more solutions. For closed paths, this can be solved by testing whether a new solution has previously been found. For open paths, this is a serious drawback, because there is no reasonable and reliable criterion to decide when to stop seeking for more solutions.

Figure 1 shows an open path of solutions for the onedimensional case. The path starts at $\lambda=0$ and the homotopy starts tracing the path of solutions. A first solution, $x_{1}^{*}$, is found at $\lambda=1$. The path continues searching for solutions for $\lambda>1$, and returns to $\lambda=1$, where a second solution, $x_{2}^{*}$, is found. This procedure is repeated for the next solutions $x_{3}^{*}$ and $x_{4}^{*}$. However, it is not possible to asses if all solutions have been found or if the procedure needs to be stopped. Most of the homotopy formulations stop seeking for more solutions after a certain number of iterations has been completed.

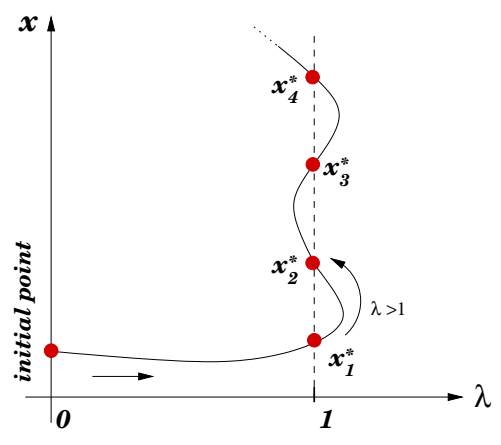

Fig. 1. Example of a solution curve

In [6], a method was proposed to limit the homotopy trajectory whithin the interval $\lambda \in[0,2]$. This method is applied under two conditions: first, it is necessary to know a priori one DC solution in order to be used as initial point 
in the method, and second, the circuit needs to be passive in the rank $\lambda \in[0,2]$. The main goal of our work is to introduce a method for creating a stop criterion for a homotopy that can be applied to general nonlinear circuits.

\section{The Doble-Bounded Homotopy}

In order to have a criterion to stop seeking for solutions, it is important to understand how to manipulate the homotopy trajectory. Usually, the homotopy finds the solutions when $\lambda$ reaches the value of 1 , but, this is merely a convention. In fact, it is possible to consider any positive value of $\lambda$ as the value where the solution is found $\left(\lambda^{*}\right)$. This allows us to introduce the concept of solution line:

Definition 1: The solution line is defined by

$$
\lambda-a=0
$$

where $\lambda$ is the homotopy parameter and $a$ is the value of $\lambda$ where the homotopy function becomes equal to the original system equation. Figure 2 show this concept.

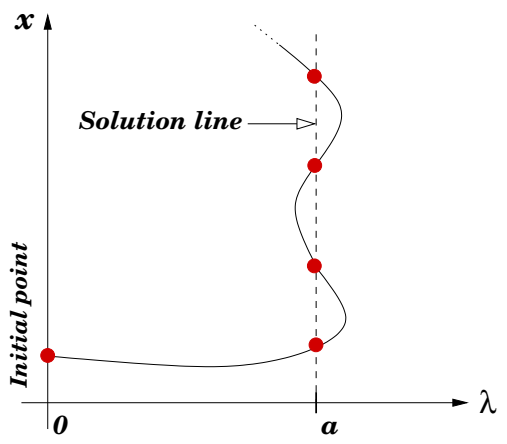

Fig. 2. The solution line

However, this scheme still shows the problems explained for Figure 1.

If the concept of one solution line is extended to twosolution lines, what will happen? There are two cases depending of number of solutions of the nonlinear system. Figure 3 depicts the case of an odd number of solutions. This figure shows an open homotopy trajectory that crosses the two solution lines $(\lambda=a$ and $\lambda=b)$ starting at $\lambda_{i}=(a+b) / 2$. The trajectory is half-bounded in the $x$-axis.

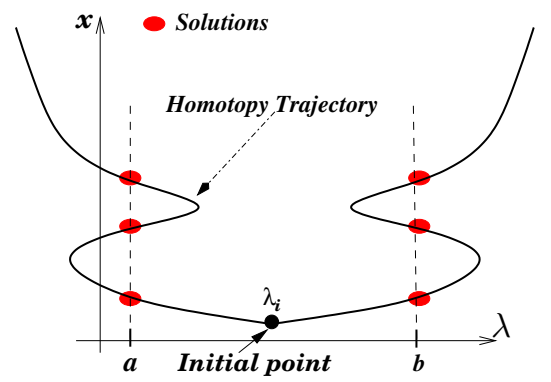

Fig. 3. Trajectory of an odd number of solutions

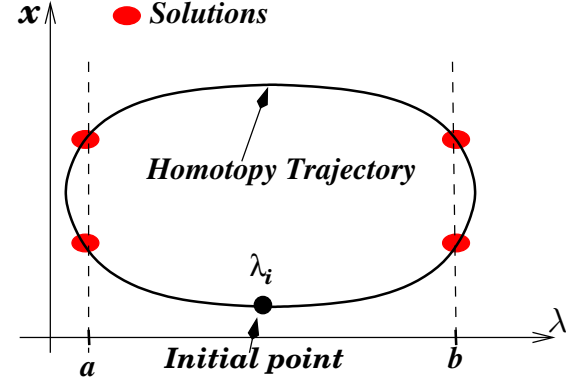

Fig. 4. A trajectory for an even number of solutions

Figure 4 depicts the behaviour of the homotopy trajectory for a case of an even number of solutions. This figure shows a closed homotopy trajectory that crosses both solution lines. These lines have the effect of modifying the homotopy trajectory. It is necessary to introduce the definition of this useful term as follows:

Definition 2: The double solution lines are defined by

$$
\lambda-a=0 \quad \text { and } \quad \lambda-b=0
$$

where $\lambda$ is the homotopy parameter, $a$ and $b$ are the values of $\lambda$ where the homotopy function becomes equal to the original system equation.

When both lines are applied to the homotopy, the result is that the trajectory is forced to cross the double solution lines. The resulting trajectory shows an interesting property, it has a symmetry axis, which splits the trajectory in two mirrored branches. The symmetry axis is defined as:

Definition 3: The symmetry axis is defined by

$$
\lambda-(a+b) / 2=0
$$

where $\lambda$ is the homotopy parameter.

Unfortunately, it is not possible to asses a priori the number of crossings on each solution line, i.e the number of solutions. Hence, a generalized stop criterion can not be established. For the case of an even number of solutions, the mirrored branches form a closed path. However, for the case of an odd number of solutions, the mirrored branches form still an open path that shows the same lacks regarding the stop criterion.

In order to circumvent this problem instead of using the original system $f(x)$, we use $f^{2}(x)$, which has the effect of converting the original $f(x)$ into an even function that is symmetric to the symmetry axis defined above, and it causes that the mirrored branches form a closed path. The results is that the number of solutions is duplicated. Figures 5(a)-(b) depict this effect with a function with two and three solutions respectively. These figures show two important properties:

1) The trajectory is closed for even and odd number of solutions.

2) The trajectory never crosses the solution lines. The solutions occur when the trajectory touches the solution lines. 


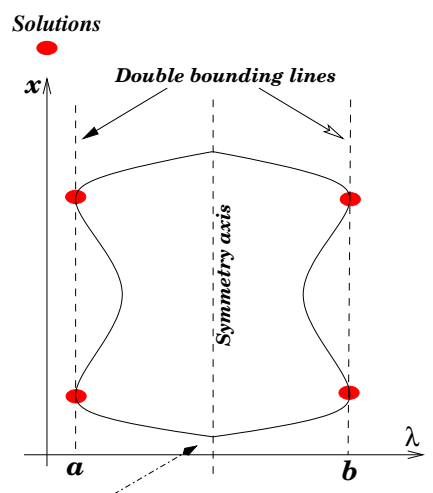

Homotopy Trajectory

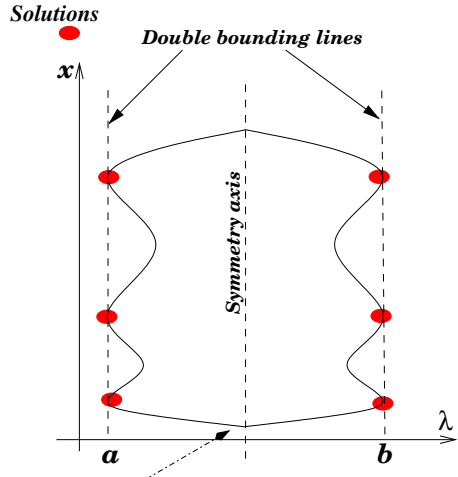

Homotopy Trajectory (a)

(b)

Fig. 5. (a) even number of solutions (b) odd number of solutions

This allows us to convert the concept of double-solution line into a pair of lines that bound the excursion of the mirrored branches. This leads to the definition of double-bounding line:

Definition 4: The double-bounding line is a special case of double-solution line and is defined by

$$
\lambda-a=0 \quad \text { and } \quad \lambda-b=0
$$

where $\lambda$ is the homotopy parameter, $a$ and $b$ are the values of $\lambda$ where the homotopy function becomes equal to the square of the original equation. Besides, the homotopy trajectory is restricted to $a \leqslant \lambda \leqslant b$

Under this scheme, we guarantee, that the bounding in the $\lambda$-direction has been achieved. However, there is still an issue regarding the values that the branches may reach in the $x$ direction. In order to have small ranges of the values in the $x$-direction, and to damp the nonlinear behavior of $f(x)$, a logarithmic term is applied to the square function.

Finally, the designed homotopy is called double-bounded homotopy and is defined as

Definition 5: The double-bounded homotopy is defined by

$$
H(f(x), \lambda)=C Q+e^{Q} \ln \left(D f^{2}(x)+1\right)
$$

where $f(x)$ is the function to solve, $\lambda$ is the homotopy parameter, $a$ and $b$ are the values of the double bounding lines, $C$ and $D$ are positive constants of the homotopy, and $Q$ is given by:

$$
(\lambda-a)(\lambda-b)
$$

The term $\left(D f^{2}(x)+1\right)$ must be used in order to assure that the argument of the log-function never becomes negative.

The symmetry axis of the double-bounded homotopy gives an important advantage over the traditional homotopies, because it can be used to shorten the lenght of the trajectory by tracing only one symmetrical branch. Figure 6 depicts the tracing strategy by shading the half side to be traced, where the initial and final points ( $x_{i}$ and $x_{f}$ respectively) of the homotopy trajectory are located at:

$$
\begin{aligned}
& \left(x_{i}, \lambda_{i}\right)=\left(x_{i},(a+b) / 2\right) \\
& \left(x_{f}, \lambda_{f}\right)=\left(x_{f},(a+b) / 2\right)
\end{aligned}
$$

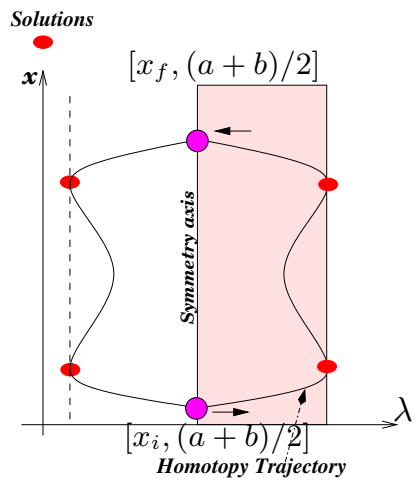

Fig. 6. Homotopy tracing based on symetrical properties

\section{A. Properties}

Herein, some properties of the double bounded homotopy are introduced.

On one side, the qualitative properties of the new homotopy are analyzed by examining the terms contained in the formulation from Equation 3. The double solution lines are contained in the next terms of the homotopy:

$$
C(\lambda-a)(\lambda-b)+e^{(\lambda-a)(\lambda-b)}(.)
$$

The double bounding effect of the new homotopy is given in the square of $f(x)$. The damping in the $x$-direction is given by the term $\ln \left(D f^{2}(x)+1\right)$.

On the other side, the properties above are explained as a result of the algebraic analysis of the homotopy formulation by resorting to the Lambert- $\mathcal{W}$ function. The main property of the Lambert- $\mathcal{W}$ function is given by [7], [8]:

$$
\mathcal{W}(z) e^{\mathcal{W}(z)}=z
$$

Firstly, Equation 3 can be reordered in the following form:

$$
-Q e^{-Q}=\frac{\ln \left(D f^{2}(x)+1\right)}{C}
$$

Now, after substituting $-Q=\mathcal{W}(z)$, we obtain:

$$
\mathcal{W}(z) e^{\mathcal{W}(z)}=\frac{\ln \left(D f^{2}(x)+1\right)}{C}
$$

Therefore, after solving for $z$, it yields:

$$
z=\frac{\ln \left(D f^{2}(x)+1\right)}{C}
$$

Therefore, $Q$ becomes:

$$
Q=-\mathcal{W}\left(\frac{\ln \left(D f^{2}(x)+1\right)}{C}\right)
$$

However, $Q$ represents the double boundings from Definition 5. After substituting in the equation above we obtain the expression:

$$
(\lambda-a)(\lambda-b)=-\mathcal{W}\left(\frac{\ln \left(D f^{2}(x)+1\right)}{C}\right)
$$


that can be solved for $\lambda$, resulting in two solutions:

$$
\begin{aligned}
& \lambda_{1}=0.5(a+b)+0.5 \sqrt{(b-a)^{2}-4 \mathcal{W}\left(\ln \left(D f^{2}(x)+1\right) / C\right)} \\
& \lambda_{2}=0.5(a+b)-0.5 \sqrt{(b-a)^{2}-4 \mathcal{W}\left(\ln \left(D f^{2}(x)+1\right) / C\right)}
\end{aligned}
$$

In fact, $\lambda_{1}$ and $\lambda_{2}$ represent the mirrored branches of the homotopy trajectory. Analysis of these expressions allows us to determine the range of values for the functions $\lambda_{1}$ and $\lambda_{2}$ :

$$
\begin{aligned}
0.5(a+b) & \leqslant \lambda_{1} \leqslant b \\
a & \leqslant \lambda_{2} \leqslant 0.5(a+b)
\end{aligned}
$$

as shown in Figure 6.

The range of the branches in the $x$-direction is defined by the function $f(x)$, when $f(x)$ satisfies:

$$
|f(x)| \leqslant(1 / D) \sqrt{D\left(e^{\left.(C / 4)(b-a)^{2} e^{(a-b)^{2} / 4}-1\right)}\right.}
$$

\section{CASE EXAMPLE}

Figure 8-a depicts the Chua's benchmark circuit having nine solutions. This circuit has 4 bipolar transistors modeled by the half-sided Ebers-Moll model (Figure 8-b).

The equilibrium equation is the same of [9] which is based on the voltages $v_{1}, v_{2}, v_{3}, v_{4}$. Figure 7 depicts the homotopy trajectory and the six solutions found versus the branch voltage $v_{1}$. The values of the found solutiones are:

$$
\begin{aligned}
& {\left[\begin{array}{l}
v_{1} \\
v_{2} \\
v_{3} \\
v_{4}
\end{array}\right]=\underbrace{\left[\begin{array}{r}
-.5136 \\
.3775 \\
-.9682 \\
.3775
\end{array}\right]}_{\text {Solution (1) }}, \underbrace{\left[\begin{array}{r}
.3242 \\
.3703 \\
-1.039 \\
.3775
\end{array}\right]}_{\text {Solution (2) }}, \underbrace{\left[\begin{array}{r}
.3869 \\
-4.632 \\
-.8002 \\
.3775
\end{array}\right]}_{\text {Solution (3) }}} \\
& \underbrace{\left[\begin{array}{r}
.3857 \\
-4.273 \\
.3322 \\
.3669
\end{array}\right]}, \underbrace{\left[\begin{array}{l}
.3300 \\
.3680 \\
.3367 \\
.3642
\end{array}\right]}, \underbrace{\left[\begin{array}{r}
-.7119 \\
.3775 \\
.3350 \\
.3653
\end{array}\right]}
\end{aligned}
$$

Solution (4) Solution (5) Solution (6)

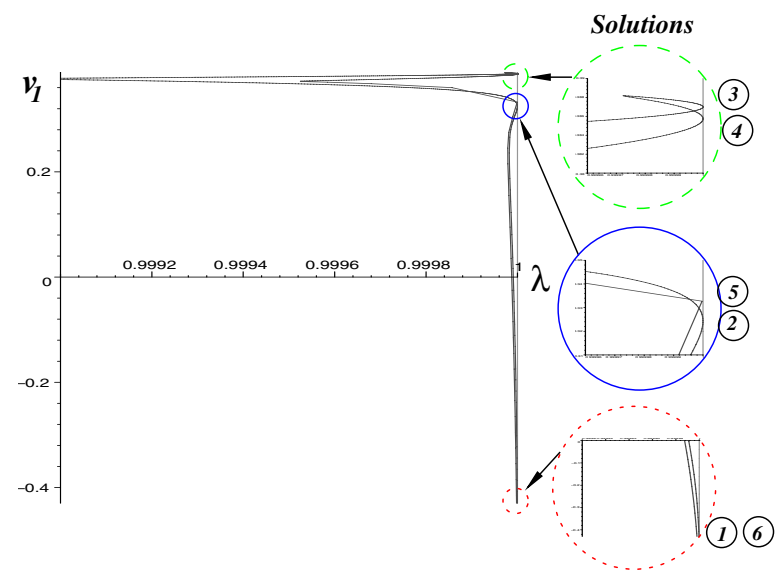

Fig. 7. Solution of the Chua's circuit

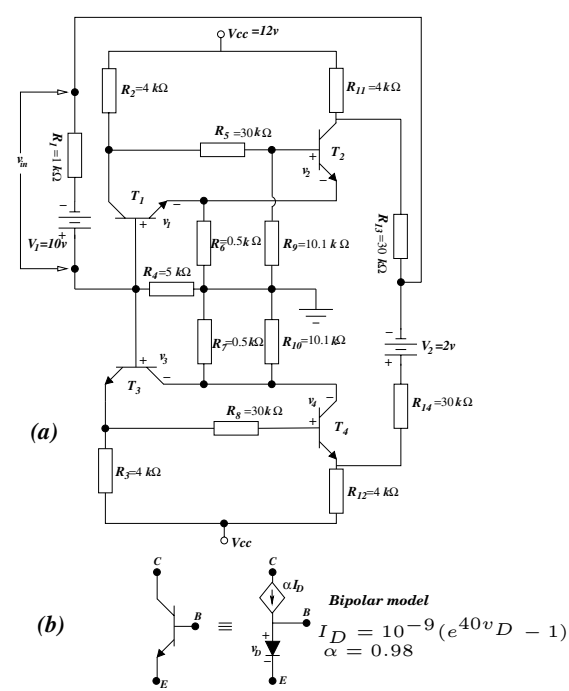

Fig. 8. Chua's circuit with nine solutions

\section{CONCLUSIONS}

A new homotopy to obtain multiple operating points of nonlinear circuits is presented. This homotopy method is called the double bounded homotopy and it overcomes the shortcomings of traditional homotopy schemes regarding the stop criterion. The geometrical properties of the method have been analyzed by using the Lambert- $\mathcal{W}$ function.

\section{ACKNOWLEDGEMENTS}

Hector Vazquez Leal is holder of a scholarship from CONACyT-México under contract 143907. This work has been partially supported by a CONACyT-México research project under grant $42588-\mathrm{Y}$.

\section{REFERENCES}

[1] J. Ogrodzki, Circuit simulation: methods and algorithms. CRC Press Inc, 1994.

[2] R. C. Melville and L. Trajković, "Artificial parameter homotopy methods for the dc operating point problem," IEEE transactions on computer-aided design of integrated circuits and systems, vol. 12, no. 6, pp. 861-877, June 1997.

[3] D. M. Wolf and S. R. Sanders, "Multiparameter homotopy methods for finding dc operating points of nonlinear circuits," IEEE transactions on circuits and systems-I: fundamental theory and aplications, vol. 43, no. 10, pp. 824-837, October 1996.

[4] K. Yamamura, T. Sekiguchi, and Y. Inuoe, "A fixed-point homotopy method for solving modified nodal equations," IEEE transactions on circuits and systems-I: fundamental theory and aplications, vol. 46, no. 6, pp. 654-664, June 1999

[5] F. H. Branin, Jr , "Widely convergent method for finding multiple solutions of simultaneous nonlinear equations," IBM Journal on Research and Dev., vol. 16, pp. 504-522, September 1972.

[6] L. B. Goldgeisser and M. M. Green, "A novel algorithm that finds multiple operating points of nonlinear circuits automatically," International Symposium on Circuit and Systems, pp. 558-561, 1998.

[7] R. M. Corless, G. H. Gonnet, D. E. G. Hare, D. J. Jeffrey, and D. E. Knuth, "On the Lambert $\mathcal{W}$ function," Advances in Computational Mathematics, vol. 5, pp. 329-359, 1996.

[8] R. M. Corless, D. J. Jeffrey, and D. E. Knuth, "A sequence of series for the lambert w function," Proceedings ISSAC, pp. 197-204, 1997.

[9] A. Ushida and L. O. Chua, "Tracing solution curves of non-linear equations with sharp turning points," Circuit Theory and Applications, vol. 12, pp. 1-21, 1984. 